\title{
SCATTERING OF SH-WAVES BY A GRIFFITH CRACK IN A LONG STRIP AT ASYMMETRIC POSITION
}

\author{
S. GHOSH, S. MANNA, AND S. C. MANDAL
}

Received 11 April 2005

The scattering of SH-waves by a Griffith crack in an infinitely long elastic strip situated at an asymmetric position has been analyzed. Applying Fourier transform, the mixed boundary value problem has been reduced to the solution of dual integral equations which finally has been reduced to the solution of a Fredholm integral equation of second kind. The numerical values of stress intensity factor, crack opening displacement, and scattered field outside the crack have been illustrated graphically to show the effect of asymmetry of the crack position.

\section{Introduction}

Cracks or inclusions are present essentially in most of the structural materials, either as natural defects or as a result of fabrication processes. The diffraction of elastic waves becomes more practical when boundaries are present in the medium. Great attention has been given to the study of diffraction of elastic waves by cracks situated at asymmetric position. Loeber and Sih [5] and Mal [6] have studied the problem of diffraction of elastic waves by a Griffith crack in an infinite medium. The problem of finite crack at the interface of two elastic half-spaces has been discussed by Srivastava et al. [11] and Boström [2]. Finite crack perpendicular to the surface of the infinitely long elastic strip has been studied by Chen [3] and by Srivastava et al. [10]. Shindo et al. [9] considered the problem of impact response of a finite crack in an orthotropic strip. Crack in inhomogeneous elastic strip has been analyzed by Sarkar et al. [8]. Matysiak and Pauk [7] studied edge crack in an elastic layer resting Winkler foundation. Recently, Birinci and Erdol [1] analyzed the problem of a layered composite containing a crack in its lower loaded-by-rigid stamp.

In our note, we have treated the diffraction of SH-waves by a crack situated at asymmetric position in an infinitely long elastic strip which has not been considered yet. This type of situation arises in almost all cases of fabrication processes in construction technology. Applying the Fourier transform, the mixed boundary value problem has been converted to the solution of dual integral equations. The dual integral equations have been finally reduced to a Fredholm integral equation of second kind by applying Abel's transform. Expressions for the stress intensity factor and crack opening displacement 
2338 Scattering of SH-waves by a crack at asymmetric position

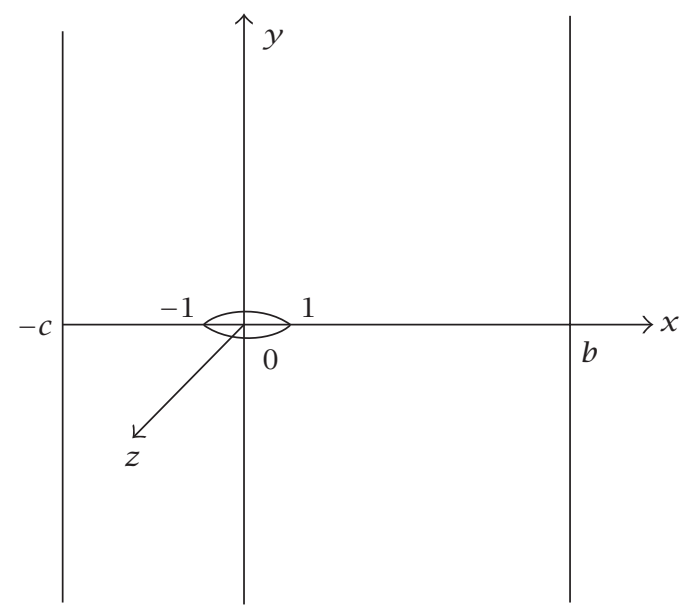

Figure 2.1. Geometry of the crack.

have been plotted to show the asymmetry of the crack position. Also stress (scattered field) outside the crack has been calculated and shown by three-dimensional graph.

\section{Formulation}

Consider the boundary value problem of interaction of SH-waves by Griffith crack situated at the asymmetric position in an infinitely long elastic strip $-d_{1} \leq x_{1} \leq d_{2},-\infty<$ $y_{1}<\infty$. The crack is located in the region $-a<x_{1}<a,-\infty<z_{1}<\infty, y_{1}=0$. Normalizing all the lengths with respect to $a$ and putting

$$
\frac{x_{1}}{a}=x, \quad \frac{y_{1}}{a}=y, \quad \frac{z_{1}}{a}=z, \quad \frac{d_{1}}{a}=c, \quad \frac{d_{2}}{a}=b,
$$

the location of the crack becomes $-1 \leq x \leq 1,-\infty<z<\infty, y=0$ (Figure 2.1) referring to a Cartesian coordinate system $(x, y, z)$. Let a plane harmonic $\mathrm{SH}$-wave originating at $y=-\infty$ impinge on crack normally to the $x$-axis. The only nonvanishing $z$-component of displacement which is independent of $z$ is $w(x, y, z)=W(x, y) e^{-i \omega t}$. Now our problem reduces to the solution of the equation

$$
\frac{\partial^{2} W}{\partial x^{2}}+\frac{\partial^{2} W}{\partial y^{2}}+k_{2}^{2} W=0
$$

where

$$
k_{2}=\frac{a \omega}{c_{2}}
$$


is subject to the boundary conditions

$$
\begin{gathered}
\tau_{y z}(x, 0)=\tau_{0} e^{-i \omega t}, \quad|x|<1, \\
W(x, 0)=0, \quad-c \leq x \leq-1,1 \leq x \leq b, \\
\tau_{x z}(-c, y)=0, \quad|y|<\infty, \\
\tau_{x z}(b, y)=0, \quad|y|<\infty .
\end{gathered}
$$

Henceforth, the time factor $e^{-i w t}$ which is common to all field variables would be omitted in the sequal.

The nonvanishing stresses are

$$
\begin{gathered}
\tau_{y z}=\mu \frac{\partial W}{\partial y}, \\
\tau_{x z}=\mu \frac{\partial W}{\partial x} .
\end{gathered}
$$

The solution of (2.2) can be taken as

$$
\begin{aligned}
W(x, y)= & \int_{-\infty}^{\infty} A(\xi) e^{-\alpha y} e^{i \xi x} d \xi \\
& +\int_{0}^{\infty}\left[B(\zeta) e^{\beta x}+C(\zeta) e^{-\beta x}\right] \sin (\zeta y) d \zeta, \quad y>0
\end{aligned}
$$

where

$$
\alpha=\sqrt{\xi^{2}-k_{2}^{2}}, \quad \beta=\sqrt{\zeta^{2}-k_{2}^{2}}
$$

Therefore the expressions of stresses are

$$
\begin{aligned}
\tau_{y z}(x, y)= & -\mu \int_{-\infty}^{\infty} \alpha A(\xi) e^{-\alpha y} e^{i \xi x} d \xi \\
& +\mu \int_{0}^{\infty} \zeta\left[B(\zeta) e^{\beta x}+C(\zeta) e^{-\beta x}\right] \cos (\zeta y) d \zeta, \\
\tau_{x z}(x, y)= & i \mu \int_{-\infty}^{\infty} \xi A(\xi) e^{-\alpha y} e^{i \xi x} d \xi \\
& +\mu \int_{0}^{\infty} \beta\left[B(\zeta) e^{\beta x}-C(\zeta) e^{-\beta x}\right] \sin (\zeta y) d \zeta .
\end{aligned}
$$


2340 Scattering of $\mathrm{SH}$-waves by a crack at asymmetric position

From the boundary conditions $(2.6)$ and $(2.7), B(\zeta)$ and $C(\zeta)$ can be found to be

$$
\begin{aligned}
& B(\zeta)=\frac{2 i \zeta}{\pi \beta\left(e^{2 b \beta}-e^{-2 c \beta}\right)}\left[e^{-c \beta} \int_{-\infty}^{\infty} \frac{\xi A(\zeta) e^{-i c \xi}}{\alpha^{2}+\zeta^{2}} d \xi-e^{b \beta} \int_{-\infty}^{\infty} \frac{\xi A(\zeta) e^{i b \xi}}{\alpha^{2}+\zeta^{2}} d \xi\right] \\
& C(\zeta)=\frac{2 i \zeta}{\pi \beta\left(e^{2 b \beta}-e^{-2 c \beta}\right)}\left[e^{c \beta} \int_{-\infty}^{\infty} \frac{\xi A(\zeta) e^{-i c \xi}}{\alpha^{2}+\zeta^{2}} d \xi-e^{-b \beta} \int_{-\infty}^{\infty} \frac{\xi A(\zeta) e^{i b \xi}}{\alpha^{2}+\zeta^{2}} d \xi\right]
\end{aligned}
$$

Now, from boundary conditions (2.4) and (2.5), we obtain the following dual integral equations for the determination of the unknown function $A(\zeta)$ :

$$
\begin{aligned}
& \int_{-\infty}^{\infty} \alpha A(\xi) e^{i \xi x} d \xi=p(x), \quad|x|<1, \\
& \int_{-\infty}^{\infty} A(\xi) e^{i \xi x} d \xi=0, \quad-c \leq x \leq-1,1 \leq x \leq b
\end{aligned}
$$

where

$$
p(x)=\frac{\tau_{0}}{\mu}+\int_{0}^{\infty} \zeta\left[B(\zeta) e^{\beta x}+C(\zeta) e^{-\beta x}\right] d \zeta .
$$

\section{Method of solution}

In order to reduce the dual integral equations (2.15) and (2.16) to a single Fredholm integral equation, we assume that

$$
A(\xi)=\frac{\tau_{0}}{2 \mu} \int_{0}^{1} \operatorname{tg}(t) J_{0}(\xi t) d t
$$

so that $(2.16)$ is automatically satisfied.

Now, (2.15) can be written as

$$
\int_{0}^{1} \xi[1+H(\xi)] A(\xi) \cos (\xi x) d \xi=\frac{p(x)}{2}, \quad|x|<1
$$

where

$$
H(\xi)=\left(\frac{\alpha}{\xi}-1\right)
$$

Substituting the value of $B(\zeta)$ and $C(\zeta)$ from (2.14) and with the help of (3.1), (3.2) can 
be converted to the following Fredholm integral equation of second kind:

$$
g(t)+\int_{0}^{1} u g(u) L(u, t) d u=1
$$

where

$$
\begin{aligned}
L(u, t) & =L_{1}(u, t)-L_{2}(u, t)-L_{3}(u, t), \\
L_{1}(u, t) & =\int_{0}^{\infty} \xi H(\xi) J_{0}(\xi u) J_{0}(\xi t) d \xi \\
L_{2}(u, t) & =\frac{1}{2} \int_{0}^{\infty} \frac{\zeta^{2} I_{0}(\beta u)\left(1+e^{-2 c \beta}\right)}{\beta\left(e^{2 b \beta}-e^{-2 c \beta}\right)}\left[I_{0}(\beta t)+L_{0}(\beta t)\right] d \zeta, \\
L_{3}(u, t) & =\frac{1}{2} \int_{0}^{\infty} \frac{\zeta^{2} I_{0}(\beta u)\left(1+e^{-2 b \beta}\right)}{\beta\left(e^{2 c \beta}-e^{-2 b \beta}\right)}\left[I_{0}(\beta t)-L_{0}(\beta t)\right] d \zeta .
\end{aligned}
$$

Using contour integration technique [11], the integral $L_{1}(u, t)$ can be converted to the following finite integral:

$$
\begin{aligned}
& L_{1}=-i k_{2}^{2} \int_{0}^{1} \sqrt{1-\eta^{2}} J_{0}\left(k_{2} \eta t\right) H_{0}^{(1)}\left(k_{2} \eta u\right) d \eta, \quad u>t, \\
& =-i k_{2}^{2} \int_{0}^{1} \sqrt{1-\eta^{2}} J_{0}\left(k_{2} \eta u\right) H_{0}^{(1)}\left(k_{2} \eta t\right) d \eta, \quad u<t .
\end{aligned}
$$

Now, putting $b=c=h$, we can find the following result for Griffith crack in a symmetric position:

$$
\begin{aligned}
L(u, t)= & -i k_{2}^{2} \int_{0}^{1} \sqrt{1-\eta^{2}} J_{0}\left(k_{2} \eta t\right) H_{0}^{(1)}\left(k_{2} \eta u\right) d \eta \\
& +\int_{0}^{\infty} \frac{\zeta^{2} I_{0}(\beta u) I_{0}(\beta t) e^{-\beta h}}{\beta \sin h(\beta h)} d \zeta .
\end{aligned}
$$

\section{Quantities of physical interest}

The shear stress $\tau_{y z}(x, y)$ in the plane $z=0$ in the neighborhood of the crack can be found from (2.12) and is given by

$$
\tau_{y z}(x, 0)=-\mu \int_{\infty}^{\infty} \alpha A(\xi) e^{i \xi x} d \xi+\mu \int_{0}^{\infty} \zeta\left[B(\zeta) e^{\beta x}+C(\zeta) e^{-\beta x}\right] d \zeta
$$

Substituting the values of $B(\zeta)$ and $C(\zeta)$ from (2.14) and using (3.1), the expression for 
2342 Scattering of $\mathrm{SH}$-waves by a crack at asymmetric position

the stress can be presented as

$$
\tau_{y z}(x, 0)=\frac{\tau_{0} x}{\sqrt{x^{2}-1}} g(1)+O(1), \quad|x|>1 .
$$

Defining the stress intensity factor $K$ by

$$
K=\lim _{x \rightarrow 1+}\left|\frac{\sqrt{x-1} \tau_{y z}(x, 0)}{\tau_{0}}\right|,
$$

we obtain

$$
K=\frac{1}{\sqrt{2}} g(1)
$$

Now, crack opening displacement

$$
\Delta W(x, 0)=W(x, 0+)-W(x, 0-)
$$

can be obtained from (2.10) as

$$
\Delta W(x, 0)=2 \int_{-\infty}^{\infty} A(\xi) e^{i \xi x} d \xi, \quad|x|<1,
$$

which on substitution of the value of $A(\xi)$ from (3.1) takes the form

$$
\Delta W(x, 0)=\frac{2 \tau_{0} a}{\mu} \int_{x}^{1} \frac{\operatorname{tg}(t)}{\sqrt{t^{2}-x^{2}}} d t, \quad|x|<1 .
$$

Scattered field $\tau_{y z}(x, y)$ for $x>1, z>0$ is calculated from (2.12), (2.14), and (3.1) and is represented by the expression

$$
\begin{aligned}
\tau_{y z}(x, y)= & -\tau_{0} \int_{0}^{\infty} \int_{0}^{1} \alpha \operatorname{tg}(t) J_{0}(\xi t) e^{-\alpha y} \cos (\xi x) d \xi d t \\
& +\tau_{0} \int_{0}^{\infty} \int_{0}^{1} \frac{\zeta^{2}\left(2 e^{-2 m \beta}+e^{-2 b \beta}+e^{-2 c \beta}\right) \cos (\zeta y) e^{\beta x}}{\beta\left(1-e^{-2 m \beta}\right)} I_{0}(t \beta) \operatorname{tg}(t) d \zeta d t,
\end{aligned}
$$

where $m=b+c$. 
S. Ghosh et al. 2343

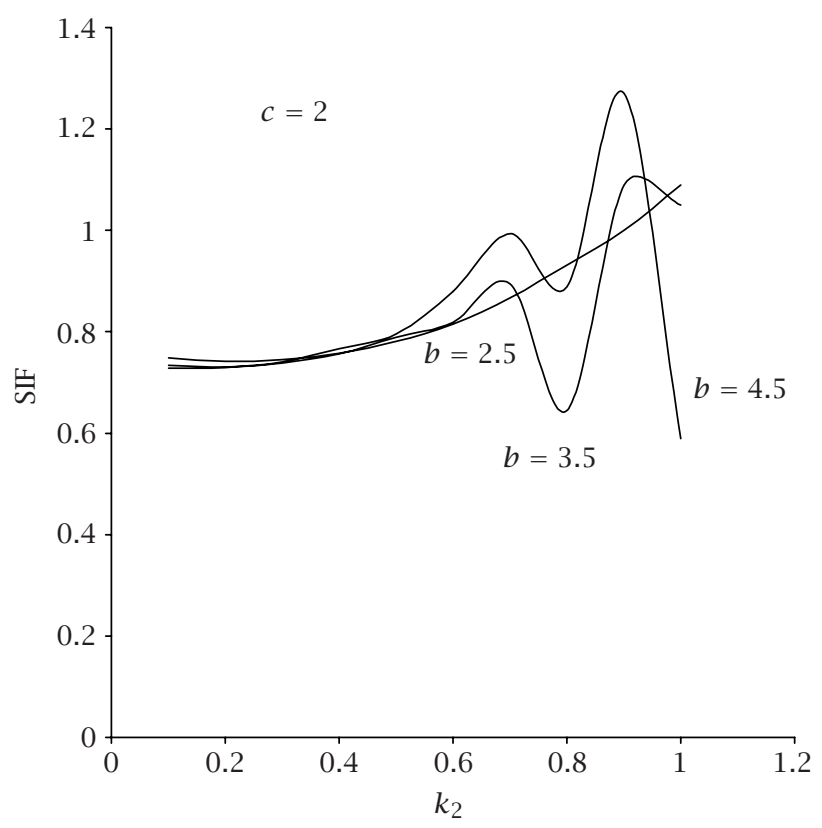

Figure 5.1. Dynamic SIF versus dimensionless frequency $k 2$.

\section{Numerical results and discussion}

The method of Fox and Goodwin [4] has been used to solve the integral equation (3.4) numerically for different values of dimensionless frequency $k_{2}$ and $b, c$ which indicate the asymmetry in position of the crack. The integral in (3.4) has been represented by a quadrature formula involving values of the desired function $g(t)$ at pivotal points inside the specified range of integration, and then converted to a set of simultaneous linear algebraic equations. The solution of linear algebraic equations gives a first approximation to the pivotal values of $g(t)$, which has been improved by the use of difference-correction technique.

After solving the integral equation (3.4) numerically, the stress intensity factor (SIF) $K$ and crack opening displacement (COD) $\mu \Delta W(x, 0) / \tau_{0}$ a have been calculated numerically and plotted separately against $k_{2}$ and dimensionless distance $x(0<x<1)$, respectively, for different values of $b$ and $c$ to show the effect of positional symmetry of the crack.

In Figures 5.1 and 5.2, $\operatorname{SIF}(K)$ is plotted against $k_{2}\left(0.1 \leq k_{2} \leq 1\right)$. In Figure 5.1, the effect of positional asymmetry of the crack is shown by varying one boundary $b(=2.5,3.5$, 4.5) while the other boundary $c(=2)$ is kept fixed and in Figure 5.2, same effect has been shown by varying $c(=1.5,2.5,3.5)$ with $b=2$. In both cases, it is observed that the SIF is increasing initially and then slowly decreasing near $k_{2}=1$ for less asymmetry in position and shows wave-like nature, and finally decreasing near $k_{2}=1$ as asymmetry in position increases.

In Figures 5.3 and 5.4, COD is plotted against $x(0 \leq x \leq 1)$ for different values of $b$, $c$, and $k_{2}$. In both cases, it is clear that COD increases as $k_{2}(=0.5,1,1.5)$ increases and 
2344 Scattering of $\mathrm{SH}$-waves by a crack at asymmetric position

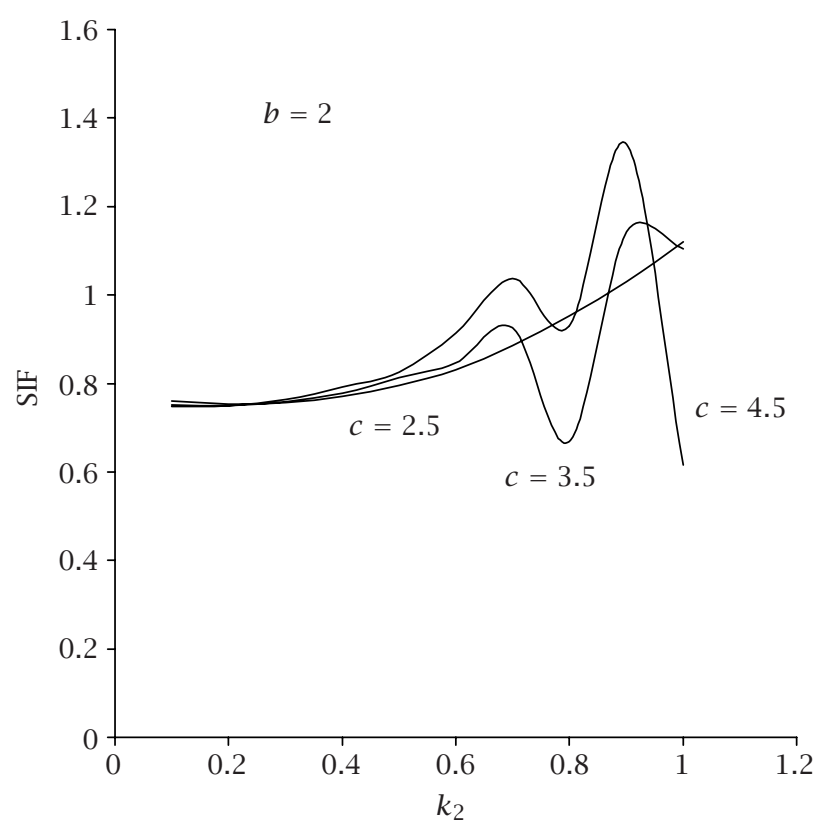

Figure 5.2. Dynamic SIF versus dimensionless frequency $k 2$.

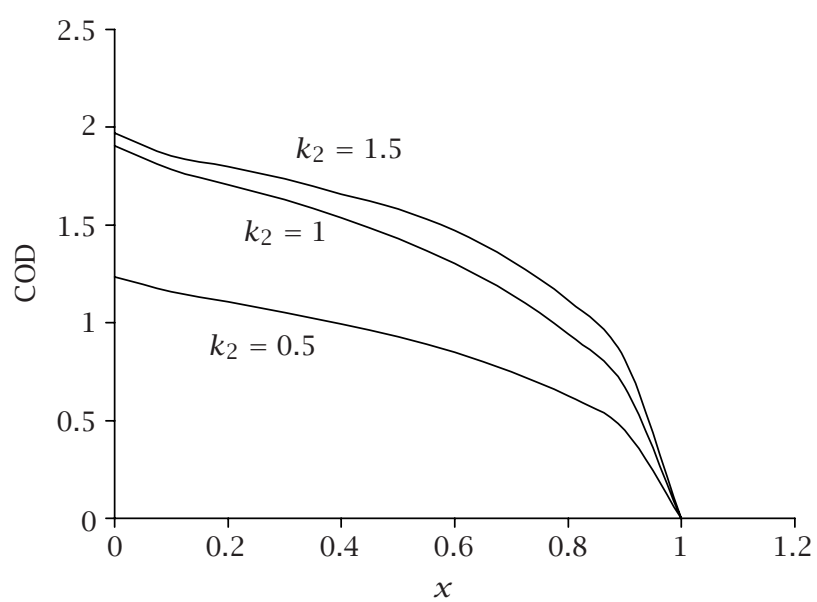

Figure 5.3. Crack opening displacement versus dimensionless distance; $b=2, c=4$.

as $x$ increases, it decreases and becomes zero at $x=1$. The maximum values of COD are at $x=0$. To calculate dimensionless scattered field $\tau_{y z}(x, y) / \tau_{0}$ outside the crack, double integrals in the expression (4.8) are evaluated for $b=4, c=3, k_{2}=4.0$ and plotted against different values of $x$ and $y$ (Figure 5.5). From Figure 5.5, it is seen that scattered field is 
S. Ghosh et al. 2345

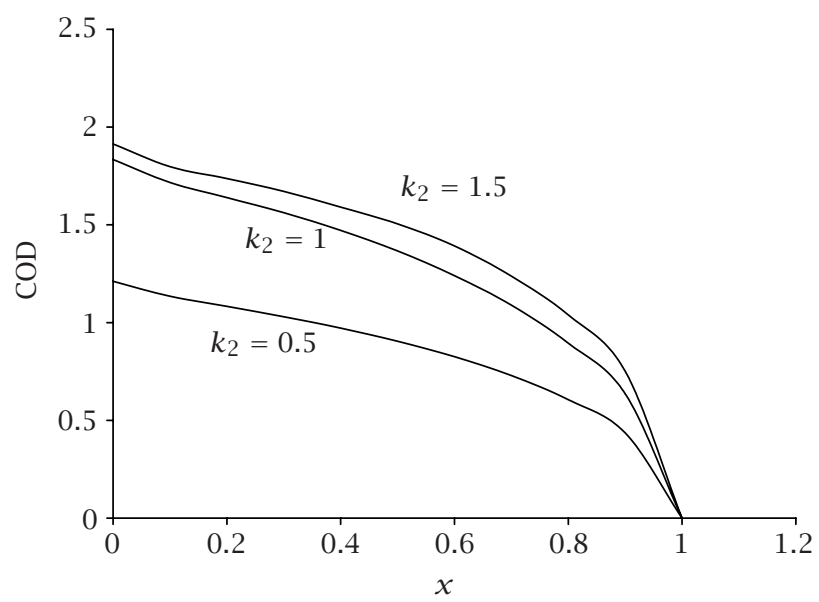

Figure 5.4. Crack opening displacement versus dimensionless distance; $b=4, c=2$.

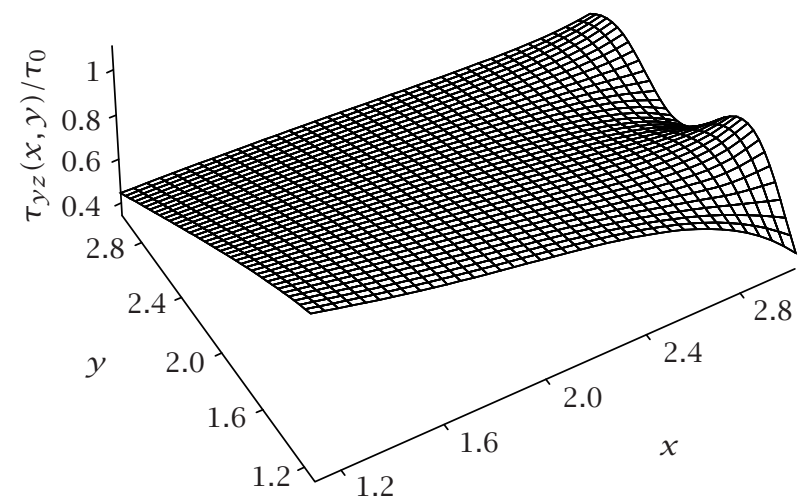

Figure 5.5. Scattered field outside the crack $\left(b=4, c=3\right.$; and $\left.k_{2}=0.4\right)$.

decreasing with increases in $x$ as well as $y$ and near boundary, it shows wave-like nature due to boundary effect.

\section{References}

[1] A. Berinci and R. Erdol, A layered composite containing a crack in its lower layer loaded by rigid stamp, Eur. J. Mech. A Solids 23 (2004), no. 6, 909-924.

[2] A. Boström, Elastic wave scattering from an interface crack: antiplane strain, J. Appl. Mech. 54 (1987), 503-508.

[3] E. P. Chen, Sudden appearance of a crack in a stretched finite strip, J. Appl. Mech. 45 (1978), 277-280.

[4] L. Fox and E. T. Goodwin, The numerical solution of non-singular linear integral equations, Philos. Trans. Roy. Soc. London Ser. A 245 (1953), 501-534.

[5] J. F. Loeber and G. C. Sih, Diffraction of antiplane shear waves by a finite crack, J. Acoust. Soc. Amer. 44 (1960), 90-98. 
2346 Scattering of $\mathrm{SH}$-waves by a crack at asymmetric position

[6] A. K. Mal, Interaction of elastic waves with a Griffith crack, Internat. J. Engrg. Sci. 8 (1970), no. 9, 763-776.

[7] S. J. Matysiak and V. J. Pauk, Edge crack in an elastic layer resting on Winkler foundation, Engrg. Fracture Mech. 70 (2003), no. 17, 2353-2361.

[8] J. Sarkar, S. C. Mandal, and M. L. Ghosh, Diffraction of SH-waves by a Griffith crack in nonhomogeneous elastic strip, Arch. Mech. 45 (1993), no. 3, 285-294.

[9] Y. Shindo, H. Nozaki, and H. Higaki, Impact response of a finite crack in an orthotropic strip, Acta Mech. 62 (1986), 87-104.

[10] K. N. Srivastava, O. P. Gupta, and R. M. Palaiya, Interaction of elastic waves with a Griffith cracks situated in an infinitely long strip, Z. Angew. Math. Mech. 61 (1981), 583-587.

[11] K. N. Srivastava, R. M. Palayiaya, and D. S. Karaulia, Interaction of antiplane shear waves by a Griffith crack at the interface of two bounded dissimilar elastic half-spaces, Int. J. Fracture. 16 (1980), no. 4, 349-358.

S. Ghosh: Department of Mathematics, Faculty of Science, Jadavpur University, Calcutta-700 032, India

E-mail address: samit_gh@yahoo.com

S. Manna: Department of Mathematics, Faculty of Science, Jadavpur University, Calcutta-700 032, India

E-mail address: jumscm@hotmail.com

S. C. Mandal: Department of Mathematics, Faculty of Science, Jadavpur University, Calcutta-700 032, India

E-mail address: jumscm@yahoo.com 


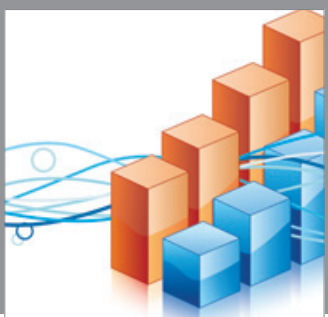

Advances in

Operations Research

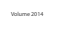

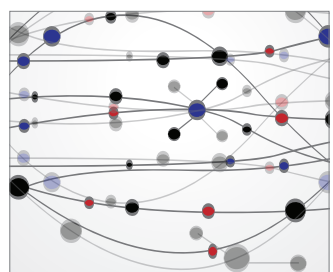

\section{The Scientific} World Journal
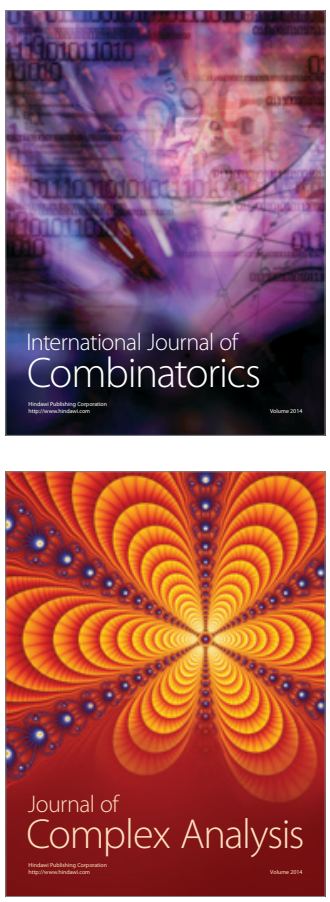

International Journal of

Mathematics and

Mathematical

Sciences
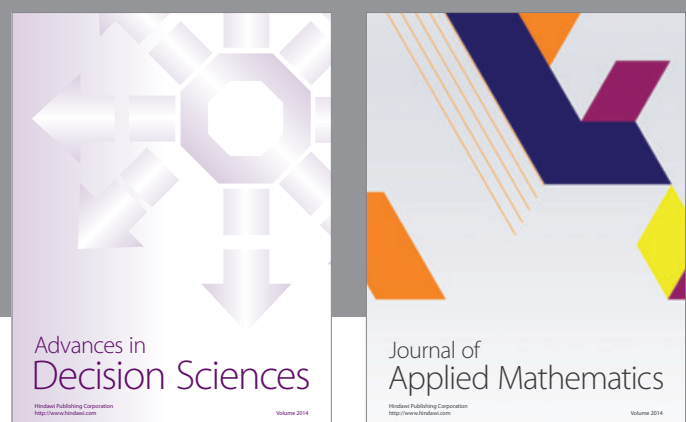

Journal of

Applied Mathematics
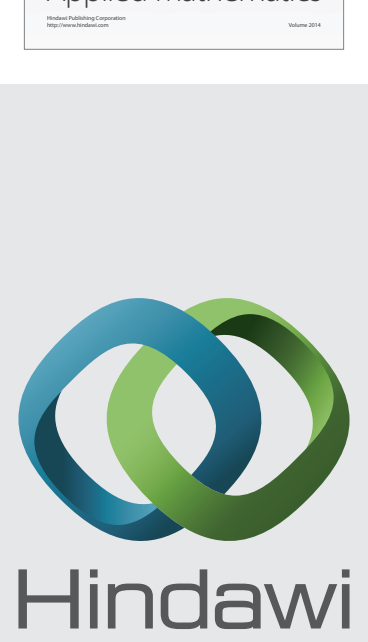

Submit your manuscripts at http://www.hindawi.com
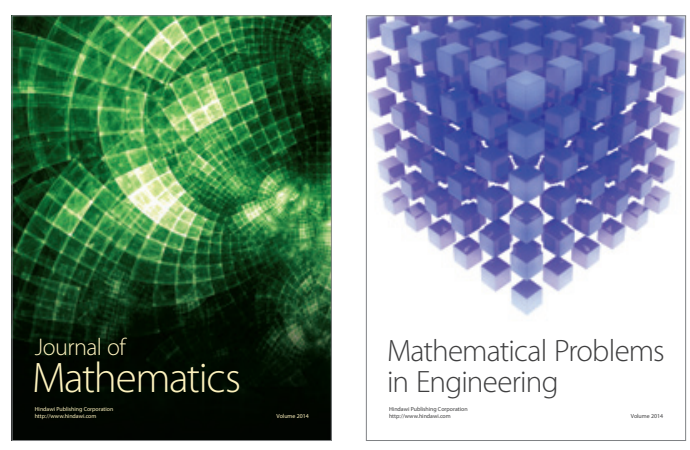

Mathematical Problems in Engineering
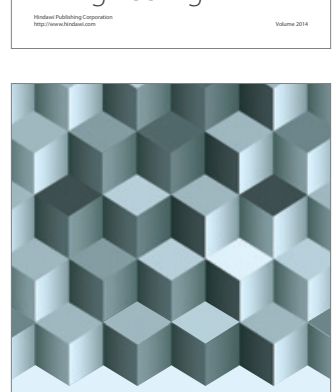

Journal of

Function Spaces
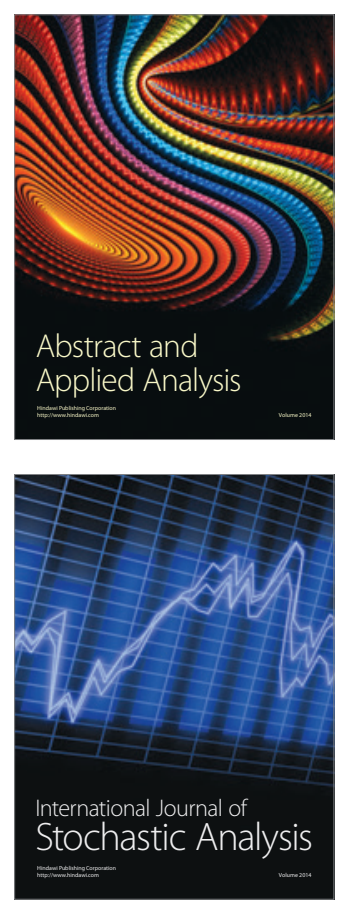

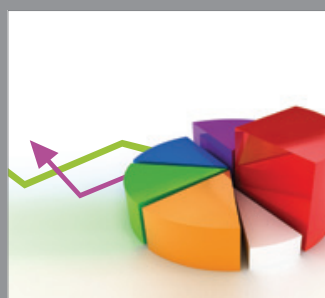

ournal of

Probability and Statistics

Promensencen
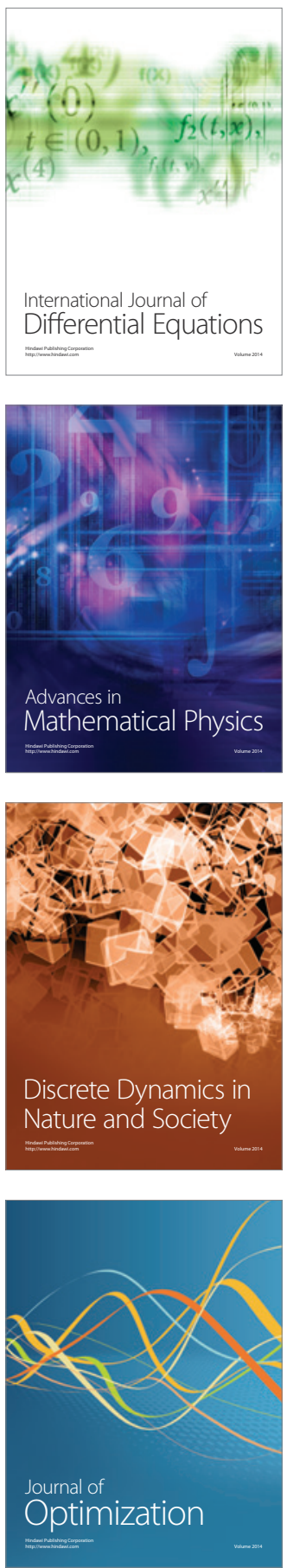